\title{
-Hairpin protein epitope mimetic technology in drug discovery
}

\author{
Obrecht, Daniel ; Chevalier, Eric ; Moehle, Kerstin ; Robinson, John A
}

\begin{abstract}
Epitopes involved in protein-protein and protein-nucleic acid interactions provide ideal starting points for rational structure-based inhibitor design. The process of design and optimization of epitope mimetics is now emerging as an innovative new approach in drug discovery. Although often derided as unsuitable for drug development, we provide examples to show how peptidomimetics can provide a new generation of drug candidates to tackle some of the most challenging targets in pharmaceutical research, and address some of the most pressing current threats to human health.
\end{abstract}

DOI: https://doi.org/10.1016/j.ddtec.2011.07.006

Posted at the Zurich Open Repository and Archive, University of Zurich

ZORA URL: https://doi.org/10.5167/uzh-69031

Journal Article

Accepted Version

Originally published at:

Obrecht, Daniel; Chevalier, Eric; Moehle, Kerstin; Robinson, John A (2012). -Hairpin protein epitope mimetic technology in drug discovery. Drug Discovery Today: Technologies, 9(1):e63-e69.

DOI: https://doi.org/10.1016/j.ddtec.2011.07.006 


\section{B-Hairpin Protein Epitope Mimetic Technology in Drug Discovery}

Daniel Obrecht ${ }^{1}$ Eric Chevalier ${ }^{1}$ Kerstin Moehle $^{2}$ and John A. Robinson ${ }^{2}$

1 Polyphor AG, Hegenheimermattweg 125, 4123-Allschwil, Switzerland (www.polyphor.com)

2 Chemistry Department, University of Zurich, Winterthurerstrasse 190, 8057-Zurich, Switzerland.

(www.oci.uzh.ch)

Corresponding author:

Prof. J. A. Robinson

Chemistry Department

University of Zurich

Winterthurerstrasse 190

8057 Zurich, Switzerland

Phone: +41-44-6354242

Fax: +41-44-6356812

e-mail: robinson@oci.uzh.ch

AND

e-mail: daniel.obrecht@polyphor.com 


\begin{abstract}
Epitopes involved in protein-protein and protein-nucleic acid interactions provide ideal starting points for rational structure-based inhibitor design. The design and optimization of epitope mimetics is now emerging as an innovative new approach in drug discovery. Although often derided as unsuitable for drug development, we provide examples to show how peptidomimetics can provide a new generation of drug candidates to tackle some of the most challenging targets in pharmaceutical research, and address some of the most pressing current threats to human health.
\end{abstract}

\title{
Introduction
}

The design of protein epitope mimetics is now recognized as a promising structure/mechanism-based approach for the discovery of protein-protein and protein-nucleic acid interaction (PPI and PNI) inhibitors. Molecular recognition involving proteins is often mediated by surface exposed secondary structure motifs such as $\beta$-hairpins and $\alpha$-helices. Interest in the development and application of new technologies to mimic such epitopes in smaller semi-rigid molecules is growing rapidly. For example, ß-hairpin motifs can be mimicked in smaller conformationally constrained macrocyclic molecules [1,2]. Such macrocycles are typically constructed from building blocks that can be linked together using robust and efficient synthetic methods. By exchanging building blocks, the mimetic structures can be varied and their properties optimized using parallel synthetic chemistry. This design and optimization process opens an area of molecular space to drug discovery, which remains difficult to explore rationally. Protein epitope mimetics are now emerging as priveleged ideal scaffolds for addressing some of the most challenging targets in drug discovery, namely, protein-protein and protein-nucleic acid interactions. 


\section{B-Hairpin mimetic design}

ß-Hairpin motifs in naturally occurring peptides and proteins possess a remarkable degree of structural diversity. The diversity can arise due to variations in the loop size, variations in the hairpin register, variations in the occurrence of $\beta$-bulges within the $\beta$-strands, and of course due to variations in sequence. It is important to note how the register of a $\beta$-hairpin defines which pairs of cross-strand amino acids occupy hydrogen-bonding (HB), rather than nonhydrogen-bonding (NHB), positions. This in turn determines which amino acid side chains are displayed on the same face of the hairpin (Figure-1A). B-Hairpin loops are prominent in the antigen-binding sites of antibodies and the ligand binding sites of many cytokine receptors and polypeptide growth factors, as well as in many integrins and viral proteins, in a wide variety of smaller host-defence peptides such as defensins, venom toxins such as $\omega$ conotoxin and three-finger snake toxins, in cyclotides, and in Bowman-Birk and related proteinase inhibitors, to name but a few.

One method to mimic a B-hairpin motif is to constrain a linear peptide using a disulfide bridge. This approach has been valuable in phage-display technology to select peptide loop structures that bind to specific protein targets [3]. A disulfide bridge, however, has disadvantages, because it can be cleaved in-vivo by reaction with a free thiol group, and the disulfide link has many degrees of rotational freedom, which in the absence of other stabilizing interactions [4], can make it difficult to stabilize discrete hairpin conformations. BHairpin conformations have been successfully stabilized in linear peptides by exploiting tryptophan zipper motifs $(\pi-\pi$-stacking interactions between cross-strand tryptophans at nonhydrogen-bonding positions) [5-7]. The residues in the turn region have a strong influence upon B-hairpin stability in linear peptides, with turns formed by Asn-Gly, D-Pro-Gly, AibGly and Aib-D-Pro being strong promoters of $\beta$-hairpin formation [8,9], due to their 
propensity to form type-I' or -II' turns. These turn types match the preferred right-handed twist of a hairpin. Other building blocks have been incorporated into turn regions and into the ß-strand regions, including for example, the 1,2-dihydro-3(6H)-pyridinyl unit that favors extended conformations [10]. Recently, a novel hairpin-capping motif has been described, which appears to overcome the problem of fraying at the ends of the $\beta$-strands in hairpin motifs in linear peptides [11]. The capping motif comprises synergistic stacking and hydrogen-bonding interactions between an N-terminal alkanoyl-Trp and a C-terminal TrpThrGly- motif, which effectively tie together the $\beta$-strands as long as the Trp residues are at terminal non-hydrogen-bonding positions.

Stable B-hairpin scaffolds can also be achieved by linking together the $\mathrm{N}$ - and $\mathrm{C}$ termini, to produce macrocyclic hairpin structures. Nature has also exploited this strategy, as evidenced by macrocyclic natural products such as gramicidin $\mathrm{S}$, which has a hairpin structure with two D-Phe-L-Pro units marking the turn regions. An interesting dependency of B-sheet periodicity on peptide length was noted in gramicidin $S$ homologs containing $6,8,10$, 12,14 and 16 residues. When the turn positions are defined at each end of the macrocycle by D-Phe-Pro, a regular hairpin structure is only seen when the connecting strands contain in total $(4 n+2)$ amino acids (where $n=$ an integer) [12]. Other approaches to macrocycles with stable parallel $\beta$-sheet scaffolds have also been described recently $[13,14]$.

An alternative approach to mimic $\beta$-hairpin structures in naturally occurring peptides and proteins, is to transplant the hairpin loop of interest onto an appropriate (semi)-rigid template (Figure-1B), or Protein Epitope Mimetic (PEM) technology [1,2]. Most success has been reported using the dipeptide fragment D-Pro-L-Pro as a template. This dipeptide fragment adopts a rigid type-II' $\beta$-turn, which is ideal to initiate anti-parallel strands held together by registered cross-strand hydrogen bonds. Upon transplanting the loop from the protein of interest, the cross-strand residue pair directly attached to the D-Pro-L-Pro template 
will then become a HB pair. In this way, accurate structural mimetics have been produced of CDR loops found in antibodies [15], of a $\beta$-hairpin loop in Tat bound to HIV-1 TAR-RNA [16-19], of protease inhibitors related to the Bowman-Birk family [20], and of cationic hostdefence peptides related to protegrin-I [21-24] and polyphemusin [25]. It is also noteworthy, that hairpin loop mimetics with quite different sizes may be designed in this way. In one case, a 13-residue disulfide cross-linked loop taken from a phage display peptide that binds a human antibody Fc fragment [26] was transplanted onto a D-Pro-L-Pro template [27]. The resulting 13-mer loop was shown to adopt a well-defined B-hairpin structure, which includes a bulge in the second strand close to the turn region, with the unusual result that side-chains of two adjacent residues point to the same side of the hairpin.

\section{Using a hairpin to mimic an $\alpha$-helix}

Interest in hairpin mimetics is enhanced further by the realization that a hairpin scaffold can be exploited to mimic naturally occurring $\alpha$-helical epitopes. Thus, the distance between the $\mathrm{C} \alpha$ atoms of two residues $i$ and $i+2$ along one strand of a hairpin is very close to that between the $\mathrm{C} \alpha$ atoms of two residues $i$ and $i+4$ on one face of an $\alpha$-helix (Figure-2). Using this information it was possible to design a $\beta$-hairpin to mimic the pharmacophore within a helical peptide derived from $\mathrm{p} 53$, which is involved in binding to its interacting protein HDM2 $[28,29]$. Template-bound $\beta$-hairpin mimetics were found that indeed bind with nanomolar affinity to HDM2. A crystal structure of one mimetic bound to HDM2 confirmed that the hairpin scaffold presents three side chains along the first strand of the hairpin for binding to HDM2, just like the corresponding three residues situated on one face of the helical p53. In addition, residues in the second $\beta$-strand of the mimetic make additional favorable contacts with HDM2, which are not seen in the p53-HDM2 complex. Other examples of hairpin mimicry of helical epitopes have been reported more recently, based on 
the interactions of Rev protein to RRE-RNA and of a helical epitope in the chemokine receptor CCR5 that binds to the HIV-1 glycoprotein gp120 [30,31]. These examples suggest that the hairpin scaffold might be widely applicable in the design of helical epitope mimetics.

\section{Hairpin mimetics in drug discovery}

Until recently, it would have been quite contentious to suggest that peptidomimetics should be considered as drug candidates. Afterall, they typically have molecular weights $>500$, possess a plethora of potential hydrogen bonding sites (i.e. they disobey the Lipinski rules [32]), are prone to be rapidly degraded in-vivo, and would in any case never cross a cell membrane. On the other hand, the genomic and proteomic revolutions continue to provide us with an ever-increasing number of mechanistic insights into biological signalling pathways, and potential targets for inhibition using small molecules. Unfortunately, many of the genes and gene products that are often most attractive from a biological perspective for targeting using small molecules are also often the least tractable from the perspective of small druglike molecules. Such targets typically include proteins that participate in PPIs or PNIs, which are often called "undruggable" within major pharmaceutical companies $[33,34]$. The difficulty in addressing "undruggable" targets using small drug-like molecules [35], however, provides a powerful motivation to explore new regions of molecular space to overcome this problem. The B-hairpin mimetics described above are positioned within a relatively unexplored region of molecular space, between that of traditional drug-like molecules and the much larger 'biologics', such as antibodies. Moreoever, recent progress suggests that many of the perceived problems associated with peptidomimetics (proteolytic stability, membrane permeability, toxicity, potency) might not be insurmountable for the goal of turning a peptidomimetic lead into a successful drug. 
In the case of the template-bound B-hairpin mimetics (PEM molecules), an important technology in the optimization of drug-like properties emerges from the field of combinatorial chemistry. These macrocyclic PEM molecules are typically constructed from building blocks that can be linked together using robust and efficient solid-phase synthesis methods. By exchanging building blocks, the mimetic structures can be quickly varied and their properties optimized, in an efficient combinatorial fashion, using parallel synthetic chemistry [36]. The ease with which PEM analogues can be produced in this way, contrasts with the difficulties that often exist in generating analogues of complex natural products. As illustrated in Figure-3, the variables for creating combinatorial libraries include the size of the B-hairpin loop, the types of amino acid building blocks, as well as the template. The proteinogenic $\alpha$-amino acids often make convenient starting points for mimetic design, but may be exchanged for any of a large number of known non-proteinogenic amino acids, or unrelated building bocks [22]. This provides great scope for optimizing drug-like properties (ADMET), as well as target affinity and specificity, within a lead series. Some recent examples that have led to clinical drug candidates are discussed below.

\section{PEM drug candidates}

\section{Serine protease inhibitors based on the Bowman-Birk motif}

The reactive site loop of the Bowman-Birk (BB) family of serine protease inhibitors comprises a ß-hairpin loop, and represents an interesting starting point for the design of PEM serine protease inhibitors. One of the smallest members of the BB family was isolated from sunflower seeds, and a crystal structure of the inhibitor bound to the active site of trypsin was taken as a starting point for PEM design [20]. Initially, mimetics were designed by transferring 11 or 7 residues from the BB reactive loop onto a D-Pro-L-Pro template. NMR studies on the resulting mimetics revealed a well-defined B-hairpin conformation in aqueous 
solution, essentially identical to that seen in the natural peptide (Figure-4A). Enzymatic assays showed that the mimetics inhibit bovine trypsin with low to mid nanomolar $\mathrm{K}_{\mathrm{i}}$ values, and an alanine scan confirmed the energetically important role of a Lys side chain, which occupies the P1 position. This PEM scaffold was recently used to generate a diverse library of serine protease inhibitors from which new, potent and selective inhibitors were discovered targeting pharmaceutically important serine proteases, such as cathepsin G, human neutrophil elastase (HNE), and tryptase, implicated in chronic obstructive pulmonary disease (COPD) and asthma [2].

For example, POL6014 is a fully reversible, competitive and potent HNE inhibitor $\left(\mathrm{K}_{\mathrm{i}} 1.4 \mathrm{nM}\right)$ with potential as an inhaled therapeutic for the treatment of lung diseases such as COPD, cystic fibrosis (CF) and acute lung injury (ALI). COPD is one of the leading causes of death and disability worldwide and there is an important medical need for new therapies that prevent disease progression or reduce mortality [37,38]. HNE plays an important role both in mucus hypersecretion and lung tissue destruction leading to emphysema [39]. A potential advantage of the PEM scaffold is that it allows formulation as an aerosol with a mass median aerodynamic diameter (MMAD) of $2.6 \mathrm{~mm}$ at the highest nebulized dose, compatible with deep lung (alveoli) respirability. In recent studies, POL6014 demonstrated a very good pharmacokinetic profile for an inhaled application in the lung. The $\mathrm{pK}$ is characterized by an excellent lung retention and a >100-fold ratio of concentrations in the airways compared to the plasma in two different species and with two different local routes of administration, thus minimizing potential side effects associated with a high systemic exposure. In addition, POL6014 was shown to significantly inhibit HNE-induced acute lung injury in mice. POL6014 was recently successfully evaluated in additional animal efficacy models, and preliminary toxicology studies in mice and monkey showed a good safety profile. 


\section{Mimetics of protegrin I as novel antibacterials}

The large family of cationic host-defence peptides plays an important role in antiinfective defence mechanisms in many organisms [40]. They are typically short cationic peptides of 10-50 residues, with a net positive charge of +2 to +10 . They often share amphipathic properties, but differ widely in sequence and secondary structures. These peptides not only possess antimicrobial activity, but also often have broad functions in the immune systems of their hosts, where they act at the interface of the innate and adaptive immune responses, and participate in multiple aspects of immunity, inflammation, wound repair and in maintaining homeostasis [41,42]. Not surprisingly, this family of peptides has already attracted great interest as a potential source of new antibiotics. Of special interest, however, are cases where novel mechanisms of antimicrobial activity can be demonstrated. In one example, the cationic antimicrobial peptide protegrin-I (PG-I) was taken as a starting point for B-hairpin mimetic design [22]. PG-I adopts a B-hairpin secondary structure in solution, which is stabilized by two cross-strand disulfide bridges. This B-hairpin is also amphipathic, so the molecule is attracted through its positive charge to the negatively charged surface of bacteria, where it can then insert into and lyse the cell membrane. PG-I displays broad spectrum antimicrobial activity, but in the micromolar range. A family of PEM molecules modeled on PG-I was reported recently, that show potent antimicrobial activity, including the lead compound L27-11 (Figure-4B) which is active in the nanomolar range, but only against Pseudomonas species and in particular the important human pathogen Pseudomonas aeruginosa [21]. The PEM molecules have a hairpin structure stabilized now by a D-Pro-L-Pro template, are positively charged and so are attracted to the surface of bacterial cells, but are not amphipathic and so do not lyse cells. Rather, they are able to target an essential bacterial ß-barrel outer membrane (OM) protein called LptD. No other peptides 
or natural products are currently known that interact with LptD (also called Imp or OstA). This OM protein forms a complex with a lipoprotein called LptE in the OM, and both are essential for the assembly of the lipopolysaccharide (LPS) layer in the outer leaflet of the OM in many Gram-negative bacteria [43-45]. When the function of LptD is blocked, OM biogenesis is disrupted. The discovery of L27-11 involved an iterative process in which an initial hit was optimized by PEM library synthesis and screening for improved antimicrobial activity. The optimal hit from each library was used as a starting point for the synthesis and testing of variations in a subsequent library. The same procedure was then followed to optimize the drug-like properties of L27-11. This included optimization of the plasma stability, target selectivity, and toxicology. This process resulted in the compound POL7001 and then a clinical lead candidate POL7080 [21]. The safety of mimetic POL7080 is now being tested in healthy humans in a phase I clinical study, but it is clear already that it represents the first in a new class of antibiotics active against Gram-negative bacteria.

\section{Inhibitors of CXCR4}

CXCR4 belongs to the class-A family of G-protein coupled receptors (GPCRs) and is used as a co-receptor for CD4-dependent HIV infection of human T-cells. Because CXCR4 is expressed on the surface of the majority of hematopoietic stem cells (HSCs) and interacts with its natural ligand SDF-1 in the bone marrow (BM), it also plays an important role in hematopoietic stem cell mobilization from the BM to peripheral blood. CXCR4 antagonists are promising new therapeutics for the efficient mobilization and harvesting of peripheral blood HSCs by disruption of the SDF-1/CXCR4 interaction [46], in cancer therapy as antimetastatic agents [47], as well as in inflammation and tissue repair [48].

Polyphemusin II is an 18-amino acid peptide isolated from the American horseshoe crab (Limulus polyphemus) that inhibits CXCR4 and adopts a $\beta$-hairpin structure in solution. 
Based on this structure, several PEM molecules were designed and optimized in biological assays. This led to highly potent and selective CXCR4 antagonist such as POL3026, POL5551 and POL6326 [2,25]. POL6326 has now successfully moved into a phase II clinical trial for autologous stem cell transplantation in newly diagnosed multiple myeloma patients. Interim results from this study show that POL6326 is safe, well tolerated and efficient in mobilizing HSCs. Grafts mobilized with POL6326 also show favourable engraftment with re-establishment of the patients' hematopoetic system. In addition, preliminary results show that POL6326 treatment mobilizes very limited or no tumor cells in the leukapheresis product. These positive findings warrant further clinical development of POL6326. In a different application, POL5551 significantly inhibited neointimal accumulation of $\mathrm{CXCR}^{+}$smooth muscle cells (SMCs) after vascular injury in a wireinduced restenosis mouse model [49]. In addition, a local lung administration of POL5551 significantly protects against inflammation and reduces lung hyper-responsiveness in an allergic murine model of asthma. These recent examples show the great therapeutic potential of PEM CXCR4 antagonists.

Recently, an X-ray structure revealed how a very closely related B-hairpin-shaped peptide binds to an engineered form of CXCR4 (Figure-4C) [50]. The ß-hairpin occupies a binding site on CXCR4 formed by residues in the inward-facing protruding walls of the seven transmembrane helical bundle, several extracellular loops, and the N-terminal segment. A network of polar, hydrogen-bonding and hydrophobic contacts between the ligand and the receptor are responsible for the specific high affinity interaction. The close structural similarity between this bound ligand and POL3026, suggets that the macrocyclic B-hairpin mimetic will interact with CXCR4 in an essentially identical manner.

\section{Conclusions}


Protein epitope mimetic technologies provide a rational approach for the design of inhibitors of some of the most challenging targets in pharmaceutical research, namely, protein-protein and protein-nucleic acid interactions. So far most effort has been on epitopes that are localized in discrete secondary structures on the surface of proteins, such as in $\alpha$-helices and B-hairpins. The macrocyclic PEM molecules highlighted in this article have the added advantage that they can be adapted to mimic $\alpha$-helical as well as hairpin epitopes. Furthermore, their production by efficient parallel synthesis methods allows the rapid synthesis and screening of libraries, and this in turn allows optimization of not only target affinity and selectivity, but also other drug-like (ADMET) properties, such as stability, pharmacology and toxicology. This has now been demonstrated in two series of $\beta$-hairpin mimetics that have each been tested in the clinic, where they have demonstrated excellent safety profiles. The two families of $\beta$-hairpin mimetics represented by POL6326 and POL7080 both target protein receptors that are located in the cell membrane. This has the added advantage that it is not a priori necessary to endow the mimetics with the property of cell permeability, which in turn is likely to pose fewer problems in toxicology. In cases where cell permeability is required, in order to address intracellular targets, methods will be required to engineer the peptide backbone, and perhaps also side chain groups, to allow passage of the mimetics across the cell membrane, by either passive diffusion or by promoted uptake. Due to their favorable physicochemical properties hairpin mimetics are also ideally suited for systemic applications (for example, by i.v., s.c., or as slow release subcutaneous implants), or alternatively for inhaled therapies (in the form of aerosols, and dry powder formulations) in the area of lung diseases. The two B-hairpin mimetics that have so far successfully entered human clinical trials (POL6326, phase II; POL7080, phase I) represent important milestones and highlight the clinical relevance of the $B$-hairpin PEM approach in drug discovey. 


\section{References}

1 Robinson, J.A. (2008) beta-Hairpin peptidomimetics: Design, structures and biological activities. Acc. Chem. Res 41 (10), 1278-1288

2 Robinson, J.A. et al. (2008) The design, structures and therapeutic potential of protein epitope mimetics. Drug Discov. Today 13 (21-22), 944-951

3 Sidhu, S.S. et al. (2000) Phage display for selection of novel binding peptides. In Meth. Enzymol. (Vol. 328), pp. 333-363, IN335, Academic Press

4 Russell, S.J. et al. (2002) Stability of cyclic B-hairpins: Asymmetric contributions from side chains of a hydrogen-bonded cross-strand residue pair. J. Am. Chem. Soc. 125 (2), 388-395

5 Cochran, A.G. et al. (2001) Tryptophan zippers: stable, monomeric ß-hairpins. Proc. Nat. Acad. Sci. USA 98, 5578-5583

$6 \mathrm{Wu}$, L. et al. (2010) Geometry and efficacy of cross-strand Trp/Trp, Trp/Tyr, and Tyr/Tyr aromatic interaction in a B-hairpin peptide. Biochemistry 49 (22), 4705-4714

7 Eidenschink, L. et al. (2009) Very short peptides with stable folds: Building on the interrelationship of Trp/Trp, Trp/cation, and Trp/backbone-amide interaction geometries. Proteins 75 (2), 308-322

$8 \quad$ Rai, R. et al. (2007) Tuning the $\beta$-turn segment in designed peptide $\beta$-hairpins: Construction of a stable type I' $\beta$-turn nucleus and hairpin-helix transition promoting segments. Pept. Sci. 88 (3), 350-361

9 Raghavender, U.S. et al. (2010) Peptide hairpin nucleation with the obligatory Type I' ß-turn Aib-DPro segment. Org. Biomol. Chem. 8 (14), 3133-3135

10 Phillips, S.T. et al. (2005) Quantifying amino acid conformational preferences and side-chain-side-chain interactions in B-hairpins. Proc. Nat. Acad. Sci. USA 102 (39), 13737-13742

11 Kier, B.L. et al. (2010) Stabilizing capping motif for B-hairpins and sheets. Proc. Nat. Acad. Sci. USA 107 (23), 10466-10471

12 Gibbs, A.C. et al. (1998) Unusual B-sheet periodicity in small cyclic peptides. Nat. Struct. Mol. Biol. 5 (4), 284-288

13 Freire, F. and Gellman, S.H. (2009) Macrocyclic design strategies for small, stable parallel B-sheet scaffolds. J. Am. Chem. Soc. 131 (23), 7970-7972

14 Woods, R.J. et al. (2007) Cyclic modular B-sheets. J. Am. Chem. Soc. 129 (9), 25482558

15 Favre, M. et al. (1999) Structural mimicry of canonical conformations in antibody hypervariable loops using cyclic peptides containing a heterochiral diproline template. J. Am. Chem. Soc. 121, 2679-2685

16 Davidson, A. et al. (2009) Simultaneous recognition of HIV-1 TAR RNA bulge and loop sequences by cyclic peptide mimics of Tat protein. Proc. Nat. Acad. Sci. USA 106, 11931-11936

17 Athanassiou, Z. et al. (2007) Structure-guided peptidomimetic design leads to nanomolar B-hairpin inhibitors of the Tat-TAR interaction of bovine immunodeficiency virus. Biochemistry 46, 741-751

18 Leeper, T.C. et al. (2005) TAR RNA Recognition by a cyclic peptidomimetic of Tat protein. Biochemistry 44, 12362-12372 
19 Athanassiou, Z. et al. (2004) Structural mimicry of retroviral Tat proteins by constrained, beta-hairpin peptidomimetics: Ligands with high affinity and selectivity for viral TAR RNA regulatory elements. J. Am. Chem. Soc. 126 (22), 6906-6913

20 Descours, A. et al. (2002) A new family of B-hairpin mimetics based on a trypsin inhibitor from sunflower seeds. ChemBioChem. 3, 318-323

21 Srinivas, N. et al. (2010) Peptidomimetic antibiotics target outer-membrane biogenesis in Pseudomonas aeruginosa. Science 327 (5968), 1010-1013

22 Robinson, J.A. et al. (2005) Properties and structure-activity studies of cyclic betahairpin peptidomimetics based on the cationic antimicrobial peptide protegrin I. Bioorg. Med. Chem. 13 (6), 2055-2064

23 Shankaramma, S.C. et al. (2003) A family of macrocyclic antibiotics with a mixed peptide-peptoid B-hairpin backbone conformation. Chem. Comm., 1842-1843

24 Shankaramma, S.C. et al. (2002) Macrocyclic hairpin mimetics of the cationic antimicrobial peptide protegrin I: A new family of broad-spectrum antibiotics. ChemBioChem. 3 (11), 1126-1133

25 DeMarco, S.J. et al. (2006) Discovery of novel, highly potent and selective betahairpin mimetic CXCR4 inhibitors with excellent anti-HIV activity and pharmacokinetic profiles. Bioorgan. Med. Chem. 14 (24), 8396-8404

26 DeLano, W.L. et al. (2000) Convergent solutions to binding at a protein-protein interface. Science 287 (5456), 1279-1283

27 Dias, R.L.A. et al. (2006) Protein ligand design: From phage display to synthetic protein epitope mimetics in human antibody Fc-binding peptidomimetics. J. Am. Chem. Soc. 128 (8), 2726-2732

28 Fasan, R. et al. (2006) Structure-activity studies in a family of beta-hairpin protein epitope mimetic inhibitors of the p53-HDM2 protein-protein interaction. Chembiochem 7 (3), 515-526

29 Fasan, R. et al. (2004) Using a beta-hairpin to mimic an alpha-helix: Cyclic peptidomimetic inhibitors of the p53-HDM2 protein-protein interaction. Angew. Chem. Int. Ed. 43 (16), 2109-2112

30 Seitz, M. et al. (2010) Peptidomimetic inhibitors targeting the CCR5-binding site on the human immunodeficiency virus type-1 gp120 glycoprotein complexed to CD4. Chem. Comm. 46 (41), 7754-7756

31 Moehle, K. et al. (2007) Design of ß-hairpin peptidomimetics that inhibit binding of alpha-helical HIV-1 Rev protein to the Rev response element RNA. Angew. Chem. Int. Ed. 46, 9101-9104

32 Oprea, T.I. et al. (2001) Is there a difference between leads and drugs? A historical perspective. J. Chem. Inform. Comp. Sci. 41 (5), 1308-1315

33 Hopkins, A.L. and Groom, C.R. (2002) The druggable genome. Nat. Revs. Drug Disc. $1,727-730$

34 Arkin, M.R. and Wells, J.A. (2004) Small-molecule inhibitors of protein-protein interactions: Progressing towards the dream. Nat. Rev. Drug Discov. 3 (4), 301-317

35 Wells, J.A. and McClendon, C.L. (2007) Reaching for high-hanging fruit in drug discovery at protein-protein interfaces. Nature 450, 1001-1009

36 Jiang, L. et al. (2000) Combinatorial biomimetic chemistry: Parallel synthesis of a small library of $\beta$-hairpin mimetics based on loop III from human platelet-derived growth factor B. Helv. Chim. Acta 83, 3097-3112

37 Mannino, D.M. and Buist, A.S. (2007) Global burden of COPD: risk factors, prevalence, and future trends. Lancet 370 (9589), 765-773

38 Barnes, P.J. (2008) Energing pharmacotherapies for COPD. Chest 134 (6), 1278-1286 
39 Barnes, P.J. (2011) Similarities and differences in inflammatory mechanisms of asthma and COPD. Breathe 7 (3), 229-238

40 Brown, K.L. and Hancock, R.E.W. (2006) Cationic host defense (antimicrobial) peptides. Curr. Opin. Immunol. 18, 24-30

41 Auvynet, C. and Rosenstein, Y. (2009) Multifunctional host defense peptides: Antimicrobial peptides, the small yet big players in innate and adaptive immunity. FEBS J. 276 (22), 6497-6508

42 Strominger, J.L. (2009) Animal antimicrobial peptides: Ancient players in innate immunity. J. Immunol. 182 (11), 6633-6634

43 Chng, S.-S. et al. (2010) Characterization of the two-protein complex in Escherichia coli responsible for lipopolysaccharide assembly at the outer membrane. Proc. Nat. Acad. Sci. USA 107 (12), 5363-5368

44 Ruiz, N. et al. (2009) Transport of lipopolysaccharide across the cell envelope: the long road of discovery. Nat. Revs. Microbiol. 7, 677-683

45 Sperandeo, P. et al. (2009) The lipopolysaccharide transport system of Gram-negative bacteria. Biochim. Biophys. Acta 1791 (Molecular and Cell Biology of Lipids), 594602

46 Jenq, R.R. and van den Brink, M.R.M. (2010) Allogeneic haematopoietic stem cell transplantation: individualized stem cell and immune therapy of cancer. Nat. Rev. Cancer 10 (3), 213-221

47 Hiratsuka, S. et al. (2011) C-X-C receptor type 4 promotes metastasis by activating p38 mitogen-activated protein kinase in myeloid differentiation antigen (Gr-1)positive cells. Proc. Nat. Acad. Sci. USA 108 (1), 302-307

48 Jujo, K. et al. (2010) CXCR4 blockade augments bone marrow progenitor cell recruitment to the neovasculature and reduces mortality after myocardial infarction. Proc. Nat. Acad. Sci. USA 107 (24), 11008-11013

49 Subramanian, P. et al. (2010) Lysophosphatidic acid receptors LPA1 and LPA3 promote CXCL12-mediated smooth muscle progenitor cell recruitment in neointima formation. Circ. Res. 107 (1), 96-105

$50 \mathrm{Wu}$, B.L. et al. (2010) Structures of the CXCR4 chemokine GPCR with smallmolecule and cyclic peptide antagonists. Science 330 (6007), 1066-1071 
Figure-1. A, Ribbon representations of two hairpin loops with different hairpin registers. The blue dotted lines represent residues at a cross-strand hydrogen-bonding (HB) position. B, In template bound hairpin mimetics the hydrogen bonding register is fixed through covalent linkage to the template (D-Pro-L-Pro, shown in purple, right). The two loop residues directly linked to the template must occupy a HB position..
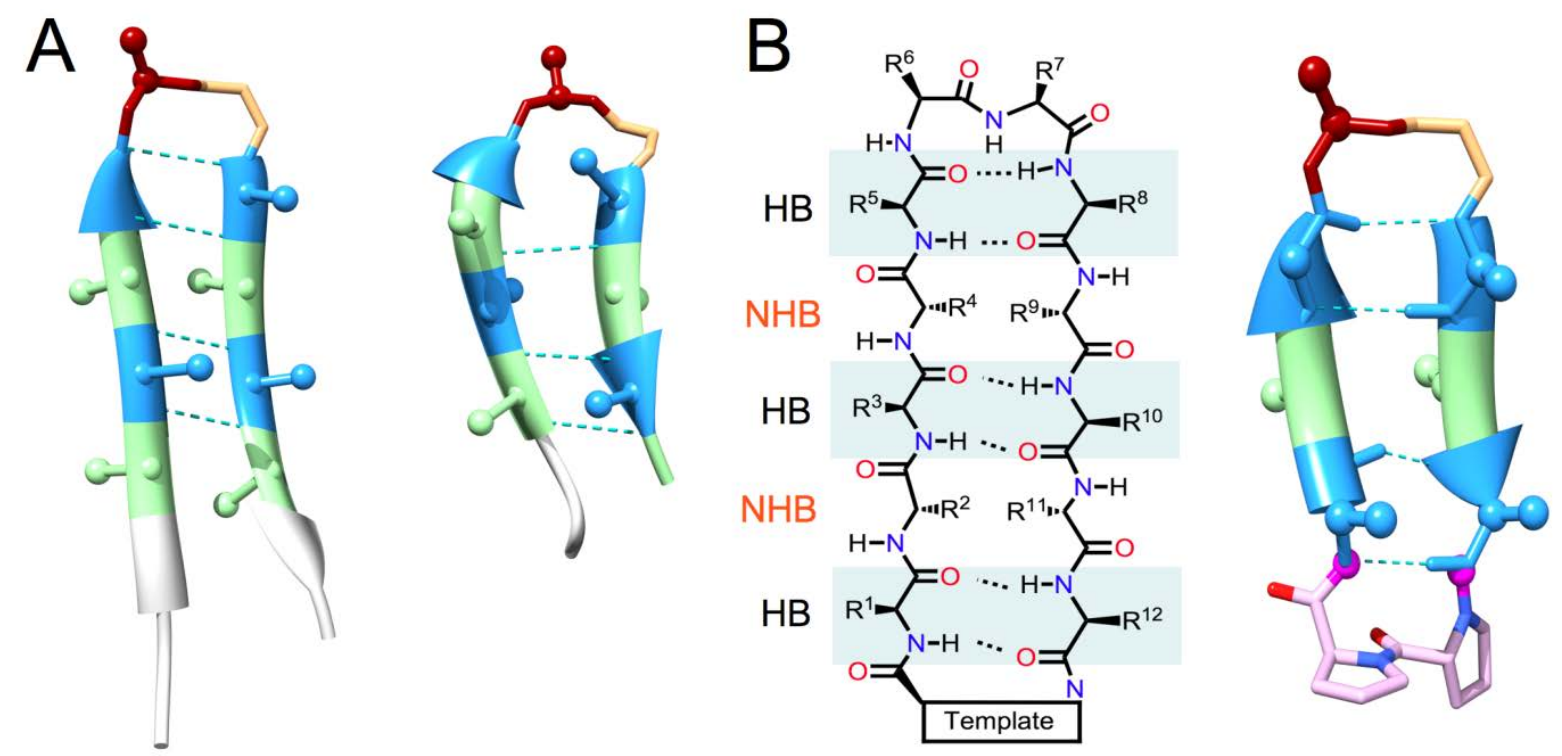
Figure-2. Superimposition of a $B$-hairpin mimetic (blue, with D-Pro-L-Pro template) and a helical epitope in p53 (pink) to illustrate how the hairpin scaffold can present three side chains equivalent to those on one face of an $\alpha$-helix (see $[28,29]$ ).

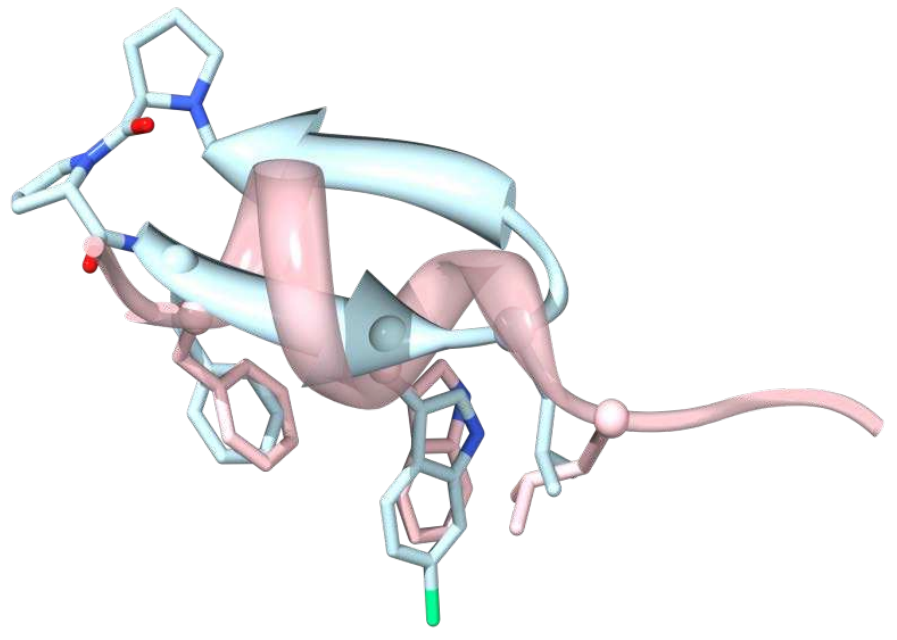


Figure-3. The synthesis of template-bound $\beta$-hairpin mimetics proceeds efficiently in a parallel combinatorial format, which allows the rapid synthesis and screening of libraries, and thereby the rapid optimization of properties.

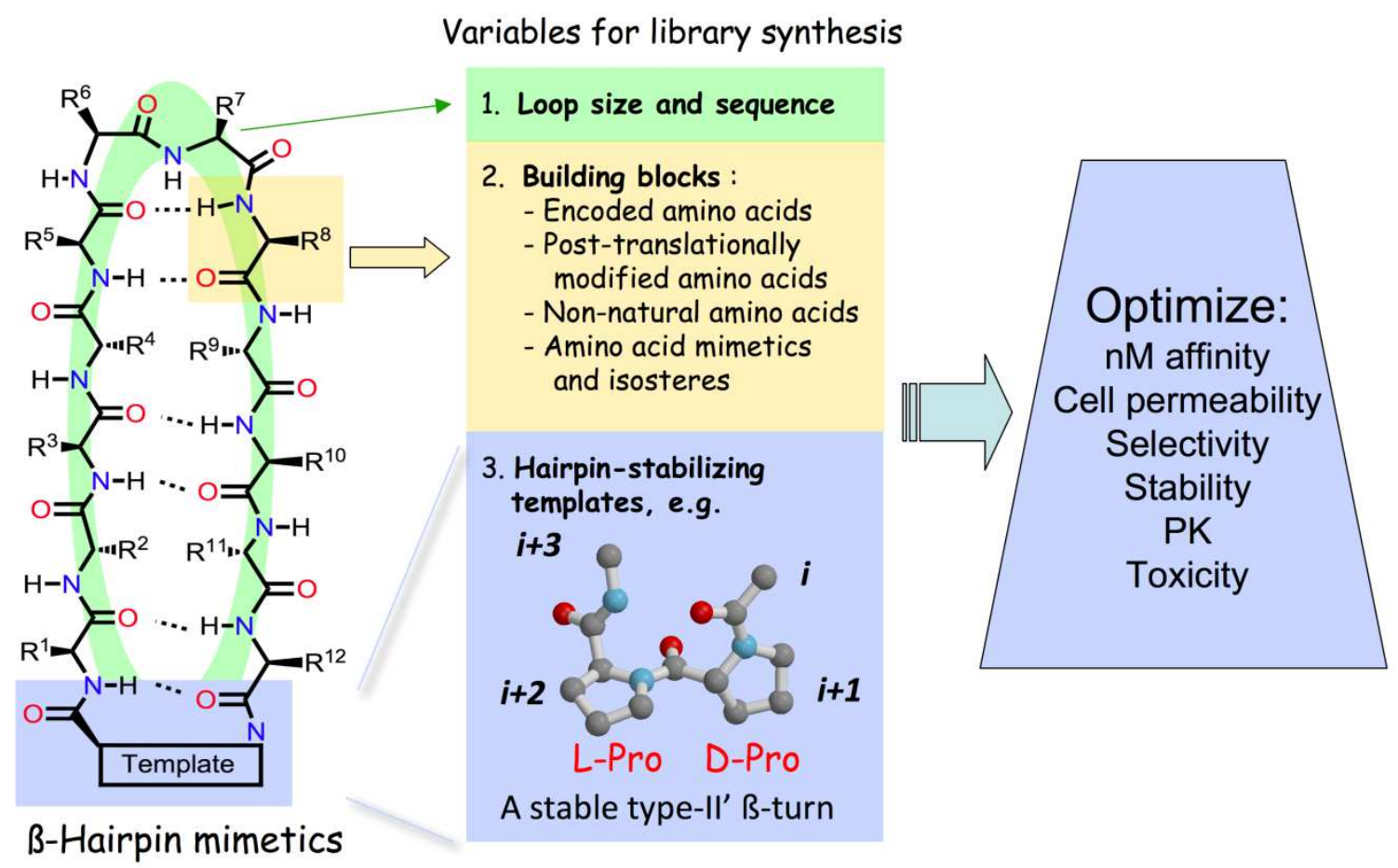


Figure-4. A, A template-bound $\beta$-hairpin mimetic (NMR structure shown in green/blue/red) superimposed upon the active-site loop of a Bowman-Birk-family protease inhibitor. $\mathbf{B}$, the template-bound B-hairpin antibiotic L27-11 that binds to the outer membrane protein LptD. C, A hairpin-shaped peptide (in green) bound to an engineered CXCR4 (PDB file 3OE0).
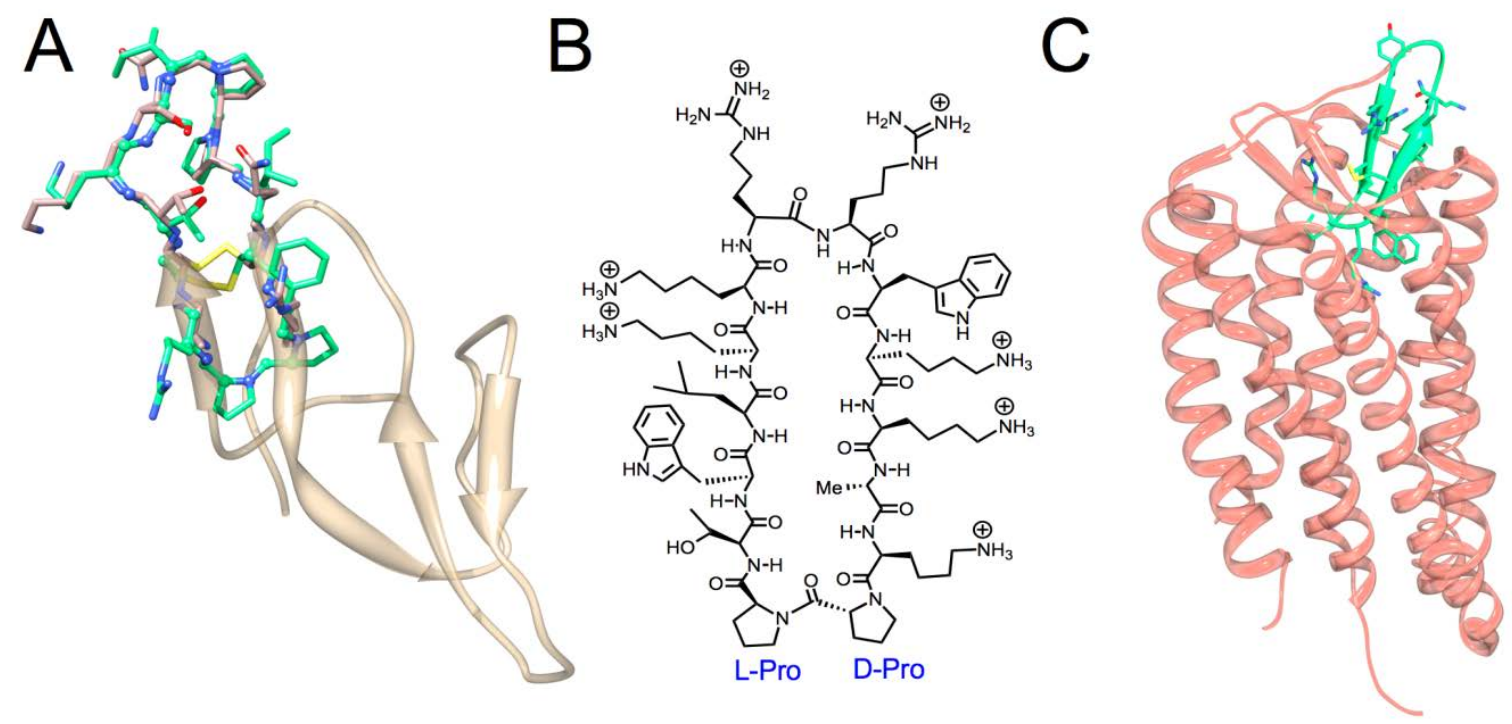